\title{
Resolving Network Issues Using Augmented Reality and Virtual Reality
}

\author{
Srilakshmi anireddy ${ }^{1}$, Archana Mantri ${ }^{2}$ \\ ${ }^{1}$ Department of Computer Science \& Engineering, Chitkara University, Punjab, India \\ ${ }^{2}$ Department of Electrical and Electronics Engineering, Chitkara University, Punjab, India \\ ${ }^{1}$ srilakshmi.anireddy@ gmail.com, 9704038864 \\ ${ }^{2}$ archanamantri.chitkara.edu.org
}

\begin{abstract}
Networking is a critical and essential commodity in today's digital world, as it establishes communication between people at remote locations. Routers, switches, cables, etc. are the essential devices of any network. Network technicians use these devices to set up the network at homes, offices and public places. In a situation of any network issue, the end-user is dependent upon the network service provider to resolve the issue as the end-user has not sufficient knowledge about the network devices. However, with the help of Augmented Reality (AR) and Virtual Reality (VR) technology, end-user can be provided with the basic training or knowledge to operate the network devices. In this paper, an AR or VR based learning environment is proposed, for providing the knowledge about different network devices and their connections. While this elementary knowledge will add to the awareness of the enduser about networking, it would also enable reducing the cost and time of the end-user and network technicians.
\end{abstract}

Keywords: Augmented Reality, Virtual Reality, Networking, Data Transmission, Network Technicians, Routers, Switch.

\section{Introduction}

In present times, the technologies available for building a fast, reliable communication network are quite advanced. The data network has thus enabled improving the quality of life of the people. People can remain connected with others, communicate, and access desired information from any part of the connected world. It is impossible to remain isolated from the internet. The internet is used to transfer the data for multiple devices. The data networks have transformed the way of interaction in social, commercial, political, and personal worlds globally. Global communities are no more dependent on location and time for their social interactions. The internet has enabled exchanging ideas and information, leading to an increase in productivity across the globe. Some forms of communication on the internet include texting, social media, blogs, collaboration tools, wikis, podcasting, and peer-to-peer file sharing [8]. Networks enable transferring the data by connecting multiple devices. It does not depend on geographical locations, physical limitations, and national borders. The network is established based on the requirement of the users. There are different types of networks with different sizes which allows us to connect two devices to millions of devices. The networks are categorised into three parts 1) Home Networks, 2) Business Networks in small and large organisations, and 3) World Wide Web. Augmented Reality (AR) and Virtual Reality (VR) are used to solve contemporary issues with the network. The contents of this paper are sequenced as follows. Section II provides a brief introduction about AR and VR. Section III explains the network requirements, types, use \& connectivity of devices, cables, switches. Section IV describes the contemporary issues solved by using AR/VR. The conclusion highlights the limitations and future scope for research

\section{Augmented Reality and Virtual Reality A.} Augmented Reality:

$\mathrm{AR}$ determines the relation between the real world and the virtual world. The computer vision technique is adapted to explain the location and orientation of the actual camera viewpoint, relative to a real-world marker. As a result, the user uses a video see-through AR on the screen or can have a more real feeling by using a headmounted device (HMD). AR technology has multiple applications across various fields, including entertainment, education, medicine, and military training, engineering, and manufacturing [5]. The AR feature recognition can be done in different ways like marker-based AR (Images, Objects), plane detection 
(horizontal, vertical), face detection and tracking [10]. The usage of the 3D environment generated by a computer is termed as Virtual Reality. VR is characterized by three elements Visualization, Immersion and Interactivity. In visualization, the user can explore by using HMD [4]. HMD tracks the user's visualizations and changes the graphics display according to view. The user navigates and interacts in the virtual world through the hardware. Reports in media, science magazines, and social fiction are some example about virtual reality. However, for the researchers involved in the science of virtual reality, the applications are much more mundane, and the problems are much more complex [3].

\section{Networks Requirements and Types}

Various devices are required to establish a network connection. The key components are Hub, Switch, Router, Bridge, Gateway, Repeater, and Cables. Hub: The device for connecting multiple networking devices is called a hub. It does not filter or address the packets but manages the data. Hubs are used to send analogue or digital data. If it receives analogue data, it passes that as a signal whereas if it receives digital data, it passes that as packets.

Switch: A multiport bridge with a buffer is called a switch. A switch is used to control the traffic by providing more number of ports. It maintains limited information about the nodes in the internal networks and performs error checking on the packets. It allows error-free packets through the network [8].

Router: A router is a device that allows the packets to reach their destination. It is similar to a switch but routes the packets based on their IP address on the packets. It maintains a routing table and connects the LAN's and WAN's. The switch is present in the data link layer, whereas the router is the device of a network layer [7].

Bridge: Bridge is used to control the flow of data Between two or more networks. It connects two LANs. It also helps to divide a large network into smaller networks. These work only at the physical layer and data link layer. Gateway: Gateways are the same as routers but have additional translation functionality. These work on the protocols and their standards [6]. The gateways are present in transport and session layers.

Cables: The different types of cables used in a network are coaxial cable, twisted-pair cable, and optical fibre cable. These cables differ with respect to their bandwidth, speed, diameter, security, price, weight, and application. The application of the cable depends on the user requirement. Coaxial cables are used in telephone networks, data networks, and where shielding of cable is necessary for protecting data from induced emf. The Twisted-pair cables are used for connection of radio transmitters and receivers with the antennas. Fiber-optics is used for high speed data over long distances across cities and countries.

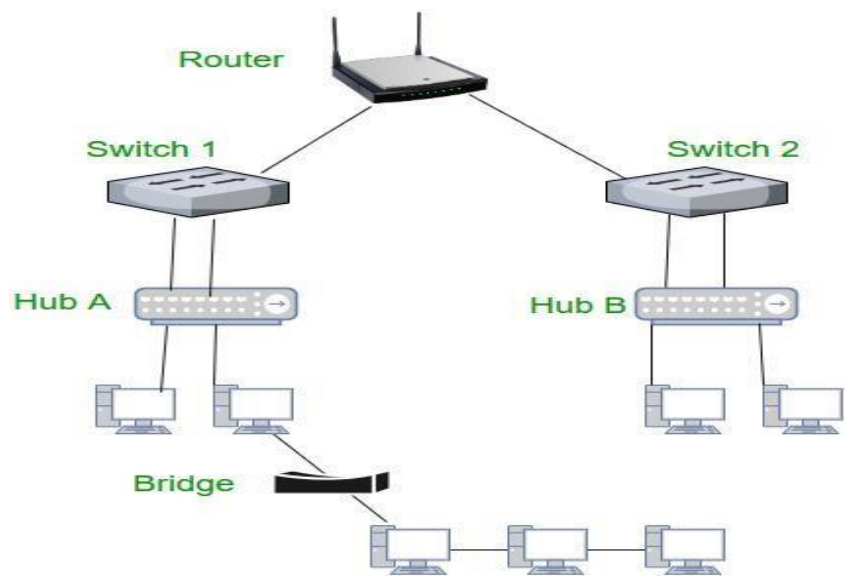

Figure 1 Example of the usage of devices

These devices are used to connect different types of networks like Local Area Network (LAN), Metropolitan Area Network (MAN), Wide Area Network (WAN), Wireless LAN (WLAN) and Storage Area Network (SAN). The selection of a network is based upon the geographical area, the number of users and types of services. LAN and WLAN are the same, and both cover the less geographical areas, for example, home networks. While LAN is a wired connection, WLAN is a wireless connection. MAN is used in business organisations; it covers the geographical area more than LAN but less than WAN. WAN is mostly used in a large city which is typically owned and managed by telecommunication service providers. Data storage and file servers are supported by SAN [9]. 


\section{Implementation of $A R / V R$ in Network Architecture}

A network used in small organisations [1] has been considered for a case study to implement AR/VR. Various devices are required to establish a network in small offices. The cost and setup this network depends upon the selection of devices [2], cables, the span of the network and the network technicians. Based on the official requirements, the network technicians established a network and explained its functioning to the users. Every organisation requires a network technician to establish a network. The lack of basic knowledge [11] of the end-users makes them dependent on network technicians for maintenance. Such dependence enhances the costs of operating and maintaining the network. As the usage of networks is expanding globally for various purposes, so the demand for network technicians is increasing. The implementation of AR/VR in networking is aimed to solve the problems of organisations and to decrease the burden of network technicians.

Every device like a router, switch, hub, etc. contains a manual with it, having step-by-step installation instructions. The network technicians have knowledge about the devices in the network, so they understand the manual for using it. In the case of misplacing/losing the manual, the network technicians can manage any device with their knowledge. So everyone depends on the network technicians installation and then its maintenance. Replacing the manual with the AR/VR can solve some maintenance issues, like 1) the functioning of the device and its operating parameters can checked/measured to decide if the device needs to be replaced or can be made good after repair, 2) showing the process of making connections of the devices in the network, and 3) providing the guidelines for replacement of any device in the network. Solv ing the above issues through an application by implementing AR/VR would reduce the cost and time of the organisations. It would also reduce the requirement of network technicians for routine issues.

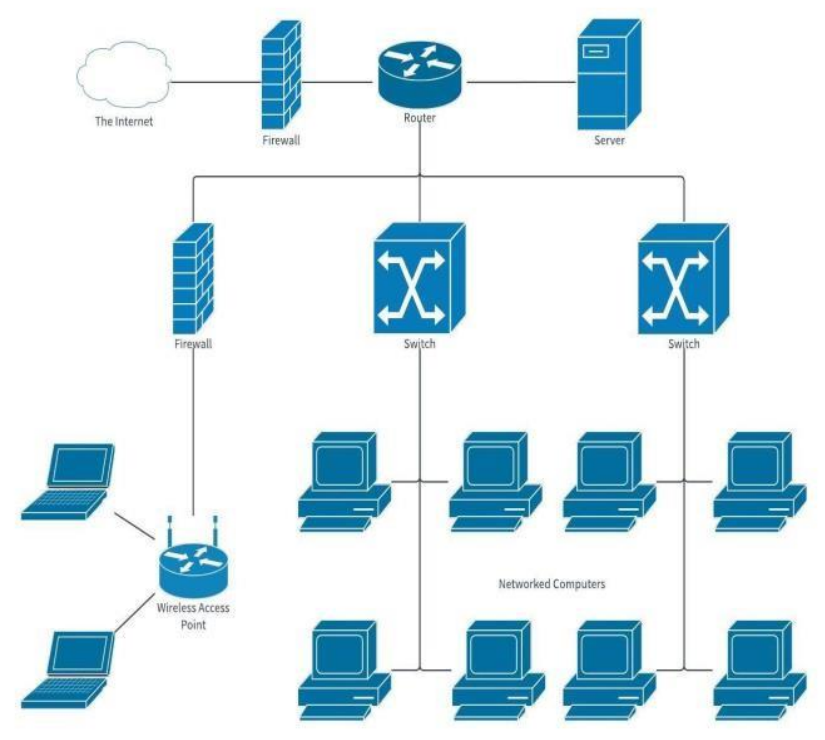

Figure 2 Small Office Network Diagram

The tools and technologies used to develop AR/VR applications are Unity, Vuforia, Blender, Headmounted-Devices and Wearable Glasses. AR is used to create a real-world environment of the network by virtual objects. It is interactive with the user and gives complete information about the devices how it is used, replaced, and connected in the network. The manual o the device is replaced by AR. By using VR, the user is immersed in the real-world environment and they can experience the connection of devices in the network and how the network works.

\section{Conclusion}

The usage of the network is increasing globally. Communication is done from remote locations through the network. The network is established based on the requirement of the user like the number of users, geographical area, and types of services. The cost and time depend on the selection of network type, usage of devices, and the number of users [2]. Enlightening the endusers with some basic knowledge on networks by using advanced technologies like AR/VR helps them to solve simple problems within their organisation. It saves the cost and time of the organisations and network administrators. This paper is limited to a single case study. It can be extended to many different types of networks. The basic problems in the home networks, in 
small and large scale organisations, can be solved and their network operation and maintenance can be simplified and made easy. The future scope is to implement an application for home network and providing the extension to many different types of networks.

\section{References}

[1] Braud, T., Bijarbooneh, F.H., Chatzopoulos, D. and Hui, P., 2017, June. Future networking challenges: The case of mobile augmented reality. In 2017 IEEE 37th International Conference on Distributed Computing Systems (ICDCS) (pp. 1796-1807). IEEE.

[2] Westphal, C., 2017. Challenges in networking to support augmented reality and virtual reality.

\section{IEEE ICNC.}

[3] Jayaram, S., Connacher, H.I. and Lyons, K.W., 1997. Virtual assembly using virtual reality techniques. Computer-aided design, 29(8), pp.575584.

[4] Yung, R. and Khoo-Lattimore, C., 2019. New realities: a systematic literature review on virtual reality and augmented reality in tourism research.

Current Issues in Tourism, 22(17), pp.2056-2081

[5] Azuma, R.T., 1997. A survey of augmented reality. Presence: Teleoperators \& Virtual Environments, 6(4), pp.355-385.

[6] Li, Z. and Li, B., 2004, September. Network coding: The case of multiple unicast sessions. In Allerton Conference on Communications (Vol. 16, No. 8).

[7] Weaver, N.S., Schallich, T.A. and Hardison, N.C., Eero LLC, 2017. Router. U.S. Patent Application 29/516,454.

[8] Smith, K.W., Ethicon Endo Surgery Inc, 2019.

Force switch. U.S. Patent 10,217,582.

[9] Hariharan, S., Loeffelholz, T. and Lumanog, G., 2018, October. Powering Outdoor Small Cells

Over Twisted Pair or Coax Cables. In 2018 IEEE
International Telecommunications Energy Conference (INTELEC) (pp. 1-6). IEEE.

[10] Katkuri, P.K., Mantri, A. and Anireddy, S., 2019, October. Innovations in Tourism Industry \& Development Using Augmented Reality (AR), Virtual Reality (VR). In TENCON 20192019 IEEE Region 10 Conference (TENCON) (pp.

2578-2581). IEEE.

[11] Janitor, J., Jakab, F. and Kniewald, K., 2010, March. Visual learning tools for teaching/learning computer networks: Cisco networking academy and packet tracer. In 2010 Sixth International Conference on Networking and Services (pp. 351355). IEEE. 